\title{
Registro Notable del Puercoespín Arborícola Sphiggurus mexicanus (Rodendia: Erethizontidae) en la Sierra Madre Oriental, México
}

\author{
Iván Lira-Torres ${ }^{1}$, Gerardo Sanchez-Rojas ${ }^{2}$, \\ Deyanira Ojeda-Ramírez ${ }^{1}$ y Fabián R. Gómez de Anda ${ }^{1}$
}

Introduction: The Mexican hairy porcupine Sphiggurus mexicanus (Rodentia: Erethizontidae) has been poorly studied in Mexico and is considered threatened according to NOM- 059- ECOL- 2010 (Mexican list of endangered species). Habitat loss due to fragmentation is a major problem for this species throughout its geographic range.

Methodology: An adult female Mexican hairy porcupine (Sphiggurus mexicanus) in good health was donated to the Nicolás Bravo Zoo in Tulancingo City, state of Hidalgo, Mexico, in June 2013. Prior to admission to the zoo, the specimen was quarantined in the veterinary school of the Universidad Autonoma del Estado de Hidalgo (UAEH).

Results: The porcupine was successfully fed a diet based on alfalfa, mango, mamey (Pouteria sapota), avocado and squash. We found two species of ectoparasites on the porcupine, Amblyomma longirostre and Trichodectes sp. The specimen of Sphiggurus mexicanus was collected at $20.29077^{\circ} \mathrm{N},-98.11047^{\circ}$ W, near the Pahuatlan River, on the border between the states of Puebla and Hidalgo, in the Eastern Sierra of Mexico.

Discussion: This location record is new and is important in that it confirms and documents the presence of the Mexican hairy porcupine Sphiggurus mexicanus, with geographical coordinates, in the Eastern Sierra of Mexico. Habitat connection to the previously known locality $70 \mathrm{~km}$ away in the area of Tuzamapan de Galeana, Puebla is not clear.

Key words: Eastern Sierra of Mexico, Mexican hairy porcupine, Sphiggurus mexicanus, records

\section{Resumen}

Se documenta el registro georeferenciado del puercoespín arborícola (Sphiggurus mexicanus) en los márgenes del río Pahuatlán en los límites entre los Estados de Puebla e Hidalgo, en la Sierra Madre Oriental. Además se informa la presencia de los siguientes ectoparásitos en el puercoespín arborícola: Amblyomma longirostre y Trichodectes sp. Este hallazgo permite registrar a la especie en una región donde había un vacio de

\footnotetext{
'Instituto de Ciencias Agropecuarias, Universidad Autónoma del Estado de Hidalgo, Área Académica de Medicina Veterinaria y Zootecnia. Rancho Universitario Av. Universidad km 1, Ex-Hacienda de Aquetzalpa. Tulancingo, Hidalgo 43600, México. E-mail: ilira_12@hotmail.com (IL), dojeda@uaeh.edu.mx (DO),mvzfabiangomez@gmail.com (FG). ${ }^{2}$ Instituto de Ciencias Básicas e Ingeniería, Universidad Autónoma del Estado de Hidalgo, Centro de Investigaciones Biológicas., Pachuca, Hidalgo, 42001, México. E-mail: gerardo.sanchezrojas@gmail.com (GS).
} 
información entre los estados de Veracruz y San Luis Potosí, por lo que se refuerza la idea de la importancia de implementar programas de monitoreo y conservación en la Sierra Madre Oriental de México.

Palabras clave: Ectoparásitos, puercoespín arborícola, registro, Sierra Madre Oriental.

Introducción

El puercoespín arborícola Sphiggurus mexicanus (Rodentia: Erethizontidae) es una de las especie de mamífero menos estudiadas de México y es considerada como amenazada de acuerdo a la NOM-059-ECOL-2010 (SEMARNAT 2010). Por lo que es de vital importancia el conocimiento de su distribución, población, ecología y demás estudios que puedan reflejar su situación actual para proporcionar información sobre su biología y así poder realizar acciones para su conservación (Juárez-G 2005).

Esta condición de riesgo es debida a la pérdida y la fragmentación de su hábitat a lo largo de su rango de distribución (Villa y Cervantes 2003). La fragmentación del hábitat se produce como consecuencia de la trasformación de los bosques tropicales en tierras agrícolas y ganaderas. No obstante, la IUCN (International Union of Conservation of Nature) considera que el estado de conservación de la especie es de preocupación menor (Pino et al. 2008). Además, no se encuentra enlistado en los Apéndices de CITES (CITES 2013).

Los puercoespines son animales arborícolas, solitarios y nocturnos, además de que pasan el día descansando en las ramas de los árboles o en algún hueco (Reid 1997; Aranda 2013). Debido a este comportamiento el registro de su presencia en alguna localidad es incidental y difícilmente se puede sistematizar (Emmons 1997; Ramírez-Bravo 2012).

La presencia de esta especie a lo largo de la Sierra Madre Oriental ha sido supuesta por métodos como los modelos de distribución potencial de especies (Pino et al. 2008), ya que se cuenta con registros referenciados geográficamente de esta especie en los estados de Veracruz y San Luis Potosí (Hall 1981; Mejenes et al. 2010; Ramírez-Bravo 2012; REMIB 2013; Fig. 1). Sin embargo, no se tiene un registro con datos geográficamente referenciados o ejemplares colectados para el norte del estado de Puebla e Hidalgo, en colecciones científicas, publicaciones, o en bases de datos electrónicas en línea (MANIS 2013; UNIBIO 2013).

Como resultado de una donación en junio del 2013, Ilego al Zoológico Nicolás Bravo en la Ciudad de Tulancingo de Bravo; en el estado de Hidalgo, un ejemplar de puercoespín arborícola (Sphiggurus mexicanus) hembra adulto en perfecto estado de salud. Previo a su ingreso a la colección animal del zoológico, el ejemplar fue puesto en cuarentena en el consultorio de Fauna Silvestre y Medicina de la Conservación del Hospital Veterinario del Área Académica de Medicina Veterinaria y Zootecnia, del Instituto de Ciencias Agropecuarias de la Universidad Autónoma del Estado de Hidalgo (UAEH), donde se evaluó y estabilizó por 40 días.

Considerando el nicho alimentario (herbívoro arborícola), se le ofreció una dieta en cautiverio a base de alfalfa fresca (Medicago sativa), mango (Mangifera sp), mamey (Pouteria sapota), aguacate (Persea americana) y chayote (Sechium edule). La dieta fue bien recibida por el ejemplar, debido a que no se presentó un desordenes gastrointestinales 
ni falta de apetito. Se le aplico un programa de medicina preventiva, que consistió en desparasitación. En el examen físico y clínico se colectaron a Amblyomma longirostre y Trichodectes sp. como ectoparásitos.

Adicionalmente y con ayuda del donante se ubicó el sitio de captura en el margen del río Pahuatlán; en donde el ejemplar fue capturado vivo, en las coordenadas $20.29077^{\circ} \mathrm{N},-98.11047^{\circ} \mathrm{W}$, en el límite entre los Estados de Puebla e Hidalgo, México (Fig. 1).

Los ecosistemas cercanos a Pahuatlán Puebla presenta muchos elementos de origen neártico. Hacia el solvento de la sierra existe presencia de remanentes de Bosques Mésofilos de Montaña representados en su mayoría por especies como pinos, encinos, liquidámbar y mixtos de carácter subhúmedo. Hacia las cañadas hay predominancia de bosques tropicales subperennifolios con predominancia de Cedrela sp., Alnus sp., Acrocarpus sp., Macademia sp., Trema sp., Inga sp., y abundantes frutales nativos (Psidium sp., Ficus sp., Pouteria sapota, etc.), como introducidos (e. g. Mangifera sp., Musa sp.), así como de vegetación secundaria y pastizales (Rzedowski 1991 González 2004). El clima es cálido húmedo con lluvias en verano (Am(f) y (A)C(w2); García 1973).

Figura.1. Mapa del sitio de captura del Puercoespín Arborícola (Sphiggurus mexicanus) en el margen del río Pahutlán, en el estado de Puebla, tanto en una escala fina que incluye los tipos de vegetación según el inventario forestal nacional, así como un mapa del lado superior derecho, donde se indican los registros reportados anteriormente de la especie para ubicar que este nuevo registro cubre un hueco sobre la información de colecta.

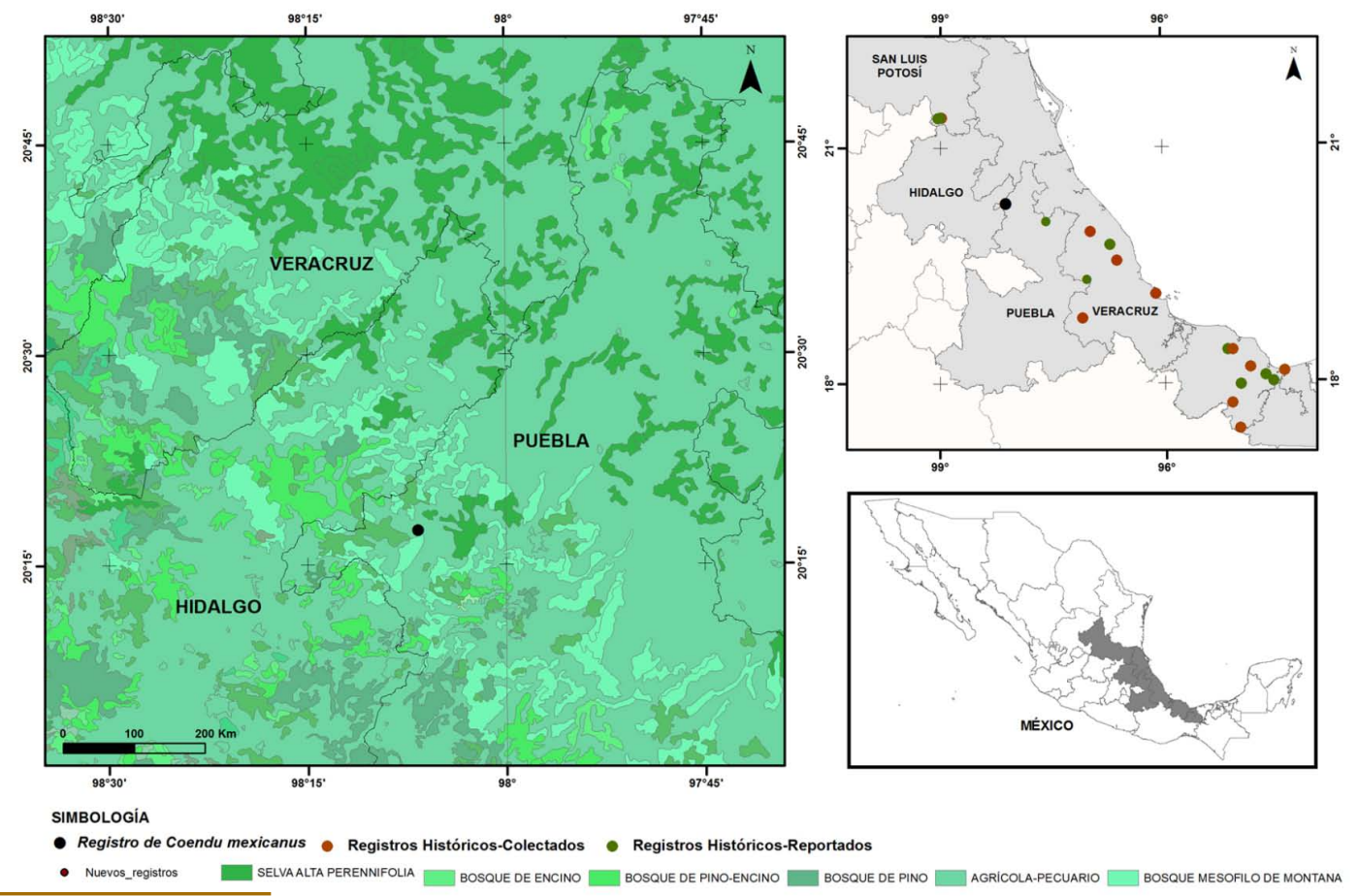

Los pobladores de Pahuatlán; próximos al sitio de colecta, del norte de Puebla, y colindante con el Estado de Hidalgo, declaran que la especie es común. Las espinas del puercoespín arborícola son buscados por la gente local, ya que se cree que son medicinales, una creencia que podría representar una amenaza para la población de puercoespines de la zona. Además, las conexiones con otras poblaciones no están claras, ya que las localidades más cercanas se encuentran a $70 \mathrm{~km}$ en el área de Tuzamapan de Galeana, Puebla y al sur de Veracruz (Tlapaya y Gallina 2010; Ramírez-Bravo 2012). Por lo que la importancia de este registro es que confirma la presencia del puercoespín arborícola Sphiggurus mexicanus en una región de la 
Sierra Madre Oriental donde existía un vacío de información sobre la presencia de la especie, el cual es muy evidente en el mapa de los registros de colecta de esta especie en las bases de datos disponibles, lo que podría ser un punto de partida para estudios dirigidos a la conservación de esta especie dentro de la zona.

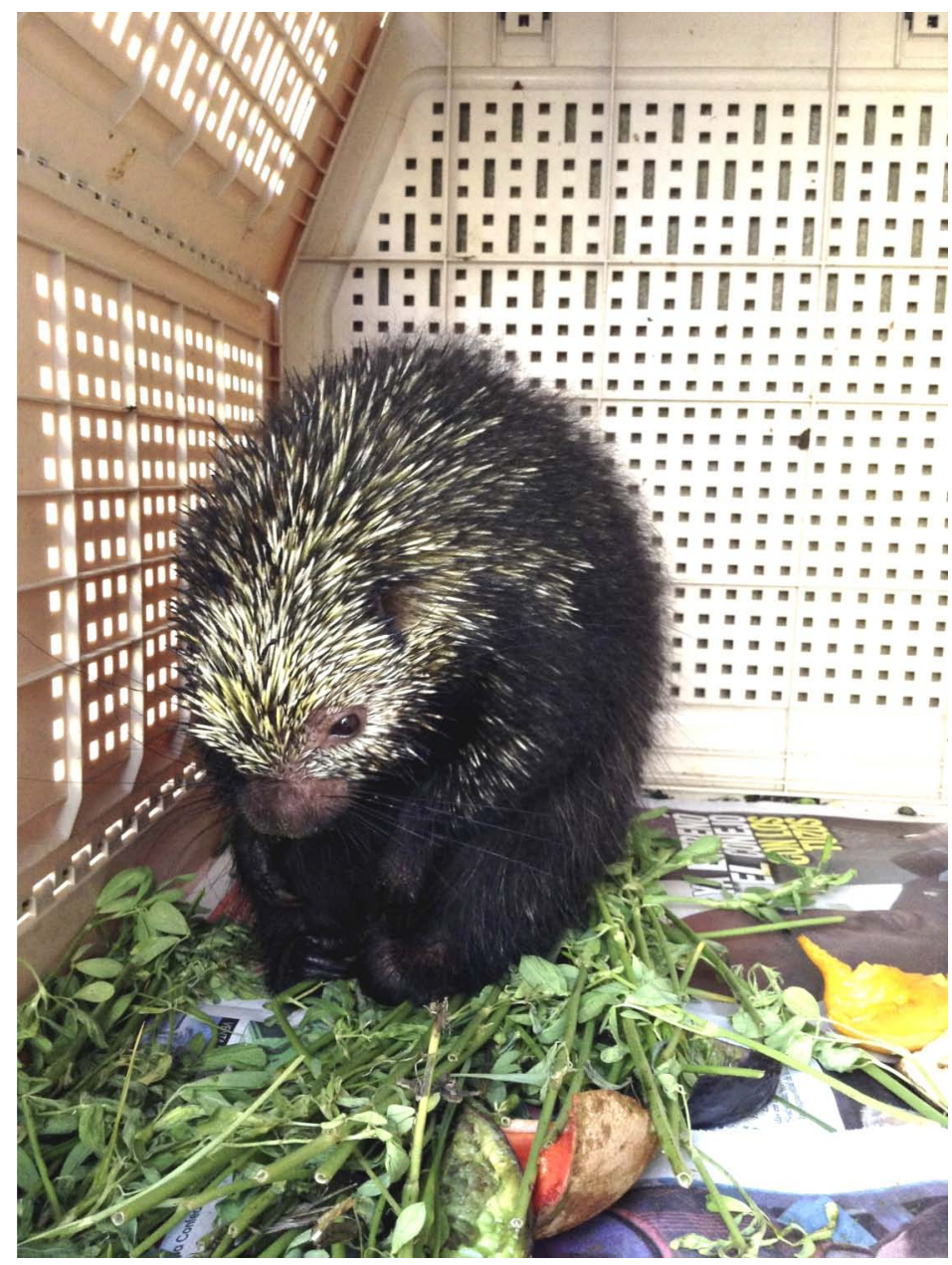

Figura 2. Ejemplar Capturado de Puercoespín Arborícola (Sphiggurus mexicanus).

Al Zoológico Nicolás Bravo de la Ciudad de Tulancingo de Bravo, Hidalgo, por Agradecimientos proporcionarnos el ejemplar y los datos de captura para la elaboración de la nota correspondiente. Agradecemos al D. A. Hernández Silva por su ayuda en el mapa de la figura 1. GSR agradece al proyecto Diversidad Biológica del estado de Hidalgo tercera etapa por el apoyo.

Aranda, M. 2012. Manual de rastreo de mamíferos silvestres de México. Comisión Nacional para el Conocimiento y Uso de la Biodiversidad (CONABIO). Ciudad de México.

Convención sobre el Comercio Internacional de Especies Amenazadas de Fauna y Flora Silvestres. 2013. URL: www.cites.org/esp/resources/pub/checklist08/index.html).

Emmons, L. 1997. Neotropical Rainforest Mammals. A Field Guide. The University of Chicago. Chicago, EE.UU. 
García, E. 1973. Modificaciones al sistema de clasificación climática de Köppen. Instituto de Geografía, Universidad Nacional Autónoma de México. Ciudad de México, México.

González, M. F. 2004. Las Comunidades Vegetales de México. Secretaria de Medio Ambiente y Recursos Naturales, Instituto Nacional de Ecología. Segunda edición. Ciudad de México, México.

HALL, R. E. 1981. The Mammals of North America. John Wiley and Sons. New York, EE.UU.

JuÁrez-G, J. 2005. Puerco espín. Pp. 811-812 in Los Mamíferos Silvestres de México (Ceballos, G., y G. Oliva, eds.) Fondo de Cultura Económica. Comisión Nacional para el Conocimiento y Uso de la Biodiversidad (CONABIO). Ciudad de México, México.

Mejenes, S. de M., M. Hernández, J. Barragán, y J. Pacheco. 2010. Los mamíferos en el Estado de Hidalgo. Therya 3:161-188.

MANIS. 2013. University of California,. http://manisnet.org/. Reviewed in 2013 August.

Pino, J., E. Vázquez, F. Reid, y A. D. Cuarón. 2008. Sphiggurus mexicanus. in: IUCN 2013. IUCN Red List of Threatened Species. Version 2013.1. <www.iucnredlist. org $>$.

Ramirez-Bravo, E. 2012. New Records of the Mexican Hairy Porcupine (Coendou mexicanus) and Tamandua (Tamandua mexicana) in Puebla, Central México. Western North American Naturalist 72:93-95.

Reid, A. F. 1997. A Field guide to the mammals of Central and Southeast Mexico. Oxford University Press. Nueva York, EE.UU.

Red Mundial de Información sobre BiodiversidAd. 2013. Comisión Nacional para el Conocimiento y Uso de la Biodiversidad. http://www.conabio.gob.mx/remib/. Reviewed in 2013 August.

Rzedowsky, J. 1991. Vegetación de México. Editorial Limusa. Ciudad de México, México.

Secretaría de Medio Ambiente, Recursos Naturales (Semarnat). 2010. Norma Oficial Mexicana NOM-059-ECOL-2010. Protección ambiental, especies de flora y fauna silvestres de México, categorías de riesgo y especificaciones para su inclusión, exclusión o cambio, y lista de especies en riesgo. Diario Oficial de la Federación, Jueves 30 de diciembre de 2010.

Unidad de Informática para la Biodiversidad-Universidad Autónoma de México. 2013. http://unibio.unam.mx/. Reviewed in 2013 August.

Tlapaya, L., y S. Galuina. 2010. Cacería de mamíferos medianos en cafetales en el centro de Veracruz, México. Acta Zoológica Mexicana (n. s.) 26:259-277.

Villa, B., y F. Cervantes. 2003. Los Mamíferos de México. Grupo Editorial Iberoamérica. Ciudad de México, México.

Sometido: 8 de septiembre de 2013

Revisado: 19 de enero de 2014

Aceptado: 5 de marzo de 2014

Editor asociado: Jan Schipper

Diseño gráfico editorial: Gerardo Hernández 
